\title{
Automatic Recognition of Features in Spectrograms Based on some Image Analysis Methods
}

\author{
Aleksandar Perović ${ }^{1}$, Zoran Đorđević ${ }^{2}$, Mira Paskota ${ }^{3}$, \\ Aleksandar Takači ${ }^{4}$, Aleksandar Jovanović ${ }^{5}$ \\ ${ }^{1}$ University of Belgrade, Faculty of Transportation and Traffic Engineering, \\ Vojvode Stepe 305, 11000 Belgrade, Serbia, pera@sf.bg.ac.rs \\ ${ }^{2}$ University of Belgrade, Faculty of Mathematics, Group for Intelligent Systems, \\ Studentski trg 16, 11000 Belgrade, Serbia, dzoran1@gmail.com \\ ${ }^{3}$ University of Belgrade, Faculty of Transportation and Traffic Engineering, \\ Vojvode Stepe 305, 11000 Belgrade, Serbia, m.paskota@sf.bg.ac.rs \\ ${ }^{4}$ University of Novi Sad, Faculty of Technology, Bulevar cara Lazara 1, 21000 \\ Novi Sad, Serbia, stakaci@tf.uns.ac.rs \\ ${ }^{5}$ University of Belgrade, Faculty of Mathematics, Group for Intelligent Systems, \\ Studentski trg 16, 11000 Belgrade, Serbia, aljosha@matf.bg.ac.rs
}

\begin{abstract}
This paper presents progress in the investigation and development of methods for the automatic localization, extraction, analysis and comparison/classification of the features in signals and their spectra. With diverse applications, different feature attributes turn out to be significant for the investigated phenomena. The general feature characteristics are morphologic and therefore suitable for a variety of algorithms focused on visual data processing, which we use in the automatic feature recognition. Our major applications were in the analysis of biological signals, and acoustic, sonar and radar signals; the methods presented here are applicable in other areas as well.
\end{abstract}

Keywords: Automatic detection of spectral features; Invariants of signal features; Brain Computer Interface; Noise elimination in radar signals

\section{Introduction}

The automatic tracking of objects represented by signals from a variety of sensors (e.g. optical, infra-red, ultra violet, sonar, radar and others) generally requires previous application of feature determination, characterization, noise reduction, background reduction and automatized extraction. Object tracking in a variety of 
sensory environments could be related to real time monitoring of the dynamic changes of their spectroscopic correlates. Among generally present signal features, some are more relevant than the others. Fourier spectroscopy with its developments and generalizations plays an important role in the investigation of signals of diverse nature. Spectral features certainly demonstrate distinguishable formations in time Fourier spectra, so spectrograms are of special value. Here, we can consider classic Fourier analysis and uniform Fourier spectroscopy based on other orthonormal systems. Their importance in the analysis of signals could be crucial, enabling accurate classification and prediction, thus providing essential insight into the properties of investigated phenomena and systems. One relatively unexplored approach to the extraction of the features from signals of various origins is based on image processing techniques applied to spectral analysis. Although different types of adaptive techniques, such as feed forward neural networks or genetic algorithms, can be applied to optimize parameters of image transformations used in this process, the goal of the presented paper is to demonstrate the feasibility of such an approach and to provide an overview of our results.

Semantically rather distant contexts often can be treated with the same or similar manners of mathematical modeling and implementations, with partial or full automatization as one of the key aims. We have selected some interesting examples of such diverse relationships from our practice. Image filtering and enhancement techniques applied to the time spectra and their further composites with well distinguished features provide automatized recognition and automatized procedures needed in various problem solutions. Common features of the spectrograms and wavelet spectrograms are aggregations that have certain topologic characteristics, like contingency, expressed boundary and differences in the intensity from its nearest neighborhood, with certain time duration. On the opposite, there are examples where distinguishable features more resemble a random dot cloud with certain density aggregation. Finally, there are short lasting features, e.g. those corresponding to the short frequency pulses in signals, which are distinguishable as dots - small sized objects in the spectrograms. All these features are often surrounded by, or embedded in, a variety of noise and artifact formations from which they need to be separated/extracted during the recognition procedures, with a degree of fuzziness present in all important properties. From the methods and algorithms we have developed and implemented, here we present ones applicable to a variety of problems in signal analysis which are related to automatic object recognition. These methods are mostly applicable in signal analysis. The software is calibrated on synthetic and alternatively measured data. Our software and signals/images together with short illustrative presentations are partially available at http://poincare.matf.bg.ac.rs/ aljosha//GIS/GIS/sbgis.htm. As illustrations we use applications in biology (e.g. signals from brain implants, EEG/MEG, neuroacoustics, blood pressure, pharmacologic applications, microscopic CCD-FISH and fNMR), acoustics and radars. This mixture of diverse examples was chosen in order to show their invariants (invariability) to problem 
unspecific applications; they just stress the kinematic and geometric equivalents of the specific system dynamics.

The recognition of spectral features in the monitoring of cardiac parameters is used to reveal an approach of a serious cardiac crisis and characterizes important states of the system and their transitions. Spectroscopy features of electro encephalography (EEG) and magneto encephalography (MEG) can help to predict epileptic seizures. It is also used in brain-computer interfaces (BCI), which has attracted our attention since the early nineties. We had available EEG recordings of externally generated tone stimulation and imagined tones and music $[1,2]$. The spectrograms of such recordings contain traces of imagined tones, which can be taken as the basis for the BCI command language. Thanks to the impressive achievements of the Rome group [3], the Graz group [4], the Tübingen group [5] and other research teams as well, BCI has become a reality recently, resulting in an explosion of interest in this area. Some researchers have suggested the use of high-frequency EEG for BCI applications $[6,7,8]$, which could expand the brain command language capacity proportionally to the increase of the speed of changes of controllable brain electro-physiological states and their number [2, 9].

\section{Method}

In automatized recognition we treat features in spectrograms, their derivates and composite spectrograms, including some real time features, with the image processing tools. The sets/manifolds in the space of $\langle$ frequency, time, intensity $>$ could possess some (simple) topological properties that are important in the characterization of investigated time spectra and related semantics, such as electrophysiology (Event-Related Synchronization (ERS) and Desynchronization (ERD) has found wide applications in the highly frequency band-specific EEG and MEG [10]), or kinematics. On the other hand, images as organized sets in the $<x, y$, intensity $>$ space often have photometric aspects that essentially characterize objects in images. We prefer to call this two-dimensional photometry a photo morphology. Hence, we have sets/manifolds in both metric spaces, time-frequency and spatial, where topologic/geometric invariants are in the focus of investigation. As we usually convert dynamics to the geometry or the other way around, we can identify those contexts when the same or similar types of invariants are used, or when we have common algorithms extracting these invariants and similarity classification. In this way, a part of the algorithm developed for the automatized analysis of morphological characteristics of, for example, chromosome and radar images, and their similarity and classification, is applicable to the study of spectroscopic features of signals, some needing adaptations and expansions. We shall illustrate such algorithms on both classes of examples, even mixing the two: the time-frequency and spatial. We discuss the implementations of the key functions involved, including algorithms which are basically simple but whose 
complexity grows with the variation of cases offered by experimental practice, thus needing increased efforts to deal with. Examples using recorded and synthetic signals have been selected to present problems, structures, methods, algorithms and solutions in a straightforward way, pointing to the aspects of interest in related problems; they all share the same corpus of analytic tools.

In order to be able to automatically detect objects in spectrograms, one first needs to separate those objects from the noise and artifacts. There are many methods for the noise removal from the signals and images, from sophisticated methods based on the Independent Component Analysis techniques to quite specialized methods we have used in the microscopic image processing, clutter in radar and sonar signals, other acoustic signal filtering, or filtering in images from cameras of different types. There are simpler and more complex situations, all requiring sophisticated noise reduction and elimination methods. In this way we have automatic noise threshold reduction, as shown in Fig. 6. However, in the case when the essential signal components are hard to separate from the noise, like when they are masked by or embedded in it, the problem of noise reduction and elimination becomes very sensitive and application dependent, with time dynamics in the noise threshold definition. Then, this step has to be subjected to a preliminary learning and intelligent treatment. The object kinetics within the observed portion of the representation space often involves object tracking as well, which, combined with the other aspects, increases problem complexity (for some examples of successful solutions of the object tracking problem, see [11, 12]). Obviously, kinetics can also be turned into a geometric form, so the tracked objects are related to their trajectories. Thus, the moving of the objects in space and the moving of the spectrogram features in time are tightly related.

We present aspects related to the feature structural morphology first, generalizing our methods for the analysis of microscopic image analysis; further on, we present adaptations of algorithms developed for the automatic object tracking and noise elimination based on the marine radar imaging.

\subsection{Aspects Related to Feature Morphology}

In Fourier spectroscopy, depending on the application, various criteria for minimum spectral stability are used, i.e. localization of sets of high/low intensity spots and lines in consecutive spectra, with topologic invariants that would distinguish them as features against e.g. smaller granular objects or random dot clouds, or all the way around. The key parameters must be determined before the application and usually involve prior knowledge of the underlying processes that are generating signals. For example, the shortest music tonal feature which is localized lasts around $0.1 \mathrm{~s}$. Before the application of any further steps, all time spectrograms need to be recalibrated, unless the time spectra were initially WYSIWYG ("what you see is what you get"), when the recalibration is not needed. Similarly, constant features need no recalibration, except for the relative 
magnitude corrections. Generally, this is a rather complex problem. In [13] is described one partial solution which introduces some time delay, which is a method for spectrogram recalibration based on the geometry of the morphology of simple features as those present in many acoustic examples. In short, the above observations form the basis for further normalizations and for the measurement of similarity with the etalon objects and posterior feature classification with the context dependent criteria. Such procedures often need to be automatized as well.

Simplified, feature localization and structure representation can be performed as follows. If we denote a spectrogram or an image in resolution $m \times n$ with $A$, then for each row $x$, first select $y_{1}$ and $y_{2}>y_{1}$ such that $A\left(x, y_{1}\right)>$ threshold and $A\left(x, y_{2}+1\right) \leq$ threshold (where threshold is a static, statistic, dynamic or learned parameter); repeating the same while $y<n$, would provide entry and exit points for each intersection of a contour with row $x$; similarly for all rows. In this way we obtain a simple localization of all contours in $A$. If artifacts are discerned by size or frequency range, they can be removed easily. After feature localization, contour stabilization-smoothing is applied if necessary, for example by removing the single convex pixels or by interpolation of the single concave pixels. Next, the topology of features is traced (Fig. 1) by two alternative processes. First, geodesy of the embedded isophotic (closed) curves determines the density gradients around feature local extremes and defines the dominant "meridian" of the feature. Alternatively, orthogonal vectors on points of the contour provide geodesic lines of the curved coordinate system, tailored to the topology of the feature. The results of the two methods are compared, providing a better contour tracing.

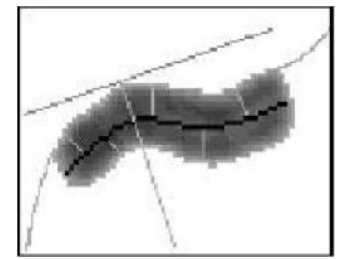

Figure 1

Feature curved coordinate system

Figure 1 shows a feature curved coordinate system tailoring and invariant capturing: positioning of the central meridian (black) and diameters perpendicular to it, determining positions of flexion points and flexion angles in the central meridian.

In the case of tonal, music or acoustic examples, the detected meridian curve possesses the information on the tonal time changes with all present dynamics (e.g. speed changes of detected vessels in submarine acoustic spectrograms, while in the case of inner music patterns or chromosomal morphologic invariants, these meridians organize the distribution of local extremes of photo density functions). Then, within tunable, reasonable approximation, the feature local extremes and 
contour local extremes are marked in the feature curved coordinate system. The central meridian is often curved and, together with flexion points and curvature in these points (the angle between the incoming and outgoing meridian fragments in the given point), presents an important feature invariant. The Fishbone demo at our web site illustrates the algorithm with normalization performed to the total feature rectification (activation of the step-by-step rectification is also available). Together with longitudinal and coordinate-wise expansion/compression factors, this can provide a good way of feature structural comparison (rectifying the corresponding features, calculating the corresponding flexure angle differences, with comparisons of corresponding segment lengths and relative positions of local extremes best fit). For the branching features, similar branch invariants could be taken into comparison considerations.

In [14] we described the similarity measures of objects in images based on geodesic (photomorphic) structural invariants (basically, the distributions of local extremes), implemented as a distance - metrics in the space of 3D manifolds. The same method can be used to characterize individual spectrogram patterns and measure their similarity using their photomorphic structural invariants. For two normalized spectrogram features $f_{1}, f_{2}$ with meridians $m_{1}$ and $m_{2}$, one way to define the morphology reflecting similarity of features, as a distance of their reasonable representations, can be

$$
d\left(f_{1}, f_{2}\right)=\min \left\{\int_{D}\left|m_{1}(x)-c \cdot m_{2}(a x+b)\right| d x: a, b, c \in \mathbb{R}\right\}
$$

where $a$ is a contraction, $b$ a translation parameter, $c$ an amplitude fitting parameter, or similar with some meridian simplifications. This also can be extended to the relative positions of local extremes, with the flexure angle differences as the second distance index. There are other ways to define combinatorial similarity of involved features, which are basically application dependent. Some examples of the real time spectrograms with the types of features we are dealing with including different common processing aspects generally applicable are shown below, with the purpose of the method details illustration. We have developed systems for the real time (RT) acquisition and real time analysis of a large number of signals.

\section{Example 1}

In Fig. 2 we present acoustic recordings suitable for automatic recognition. 

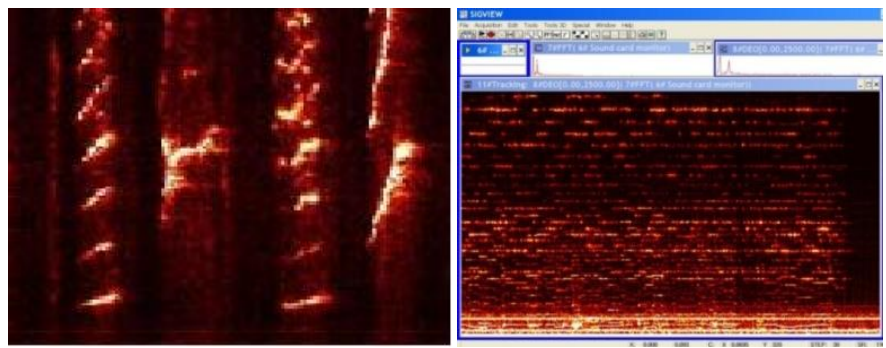

Figure 2

Examples of spectrogram features

The image on the left contains a spectrogram of a humpback whale song; the large structures correspond to melodious patterns, which are changing in both frequency and rhythm. On the right we have spectrogram of Die Kunst der Fuge, with all the tonal features of Bach's monumental music structure, in the last minute of performance by $\mathrm{H}$. Walcha. Submarine sonar spectrograms can be rather similar to the ones presented above. Clearly, in the case when the percepted and imagined music tones are the same, we should expect spectrograms of a similar type and complexity, with spectrogram features of the imagined tones and music having similar properties as those of the acoustic origin, obviously with other features corresponding to the brain activity. We can distinguish inner tones, which are stable in frequency and thus flat in spectrograms, creating strong contrasts between high $\mathrm{C}$ versus low $\mathrm{A}$ as BCI signals, and tones with variable frequencies, with a kind of linear or nonlinear frequency inclination. Such features are in fact additional specialized filters detecting salient characteristics of the signal, as for example phonemic structures are detected in speech. The segments of the spectrograms of brain signals from different experiments with BCI are shown in Figs. 3 and 4.
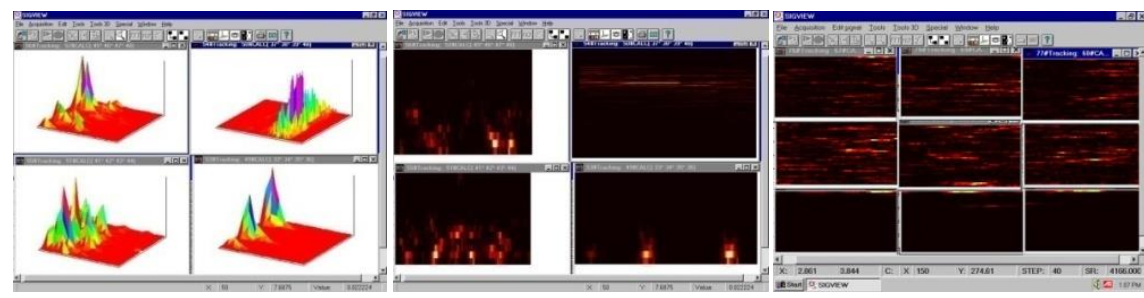

Figure 3

EEG spectral features

The left and center images in Fig. 3 show composite spectrograms of four EEG experiments exhibiting different types of imagined features prepared for automatic recognition with sporadic artifacts; the feature in the top right corner is lasting and stable in frequency, while the other three spectrograms exhibit the presence of dominant short lasting pulses; vertical - frequency range of $15 \mathrm{~Hz}$, time scale of 10 s. The right image shows the fragments of real time spectrograms of EEG, 
containing features corresponding to the traces of inner tones. Vertically frequency intervals; horizontally, time scale of 10 s. In Fig. 4, improved signal/noise provides object recognition directly, using partial linear dependence of two sources (spectrograms): the noise (random) is of a local nature while the signal components are present in both. These time spectra contain features related to imagined - inner tones, but these spectra show the presence of features corresponding to other processes in the brain as well. Searching for invariant structures in the spectrograms, we can obtain spectrograms containing only relevant features that will filter out all spectrogram structures not related to the inner tones. Before the similarity matching and measurements, in the case of inner tone classifier, we need to perform feature aggregation and disintegration. This process is application dependent: geometrically close objects should be aggregated into a single structure. On the other hand, features with a frequency inclination can be fragmented into individual tones (step functions) so that they can be subjected to matching with the set of calibration fuzzy tones.

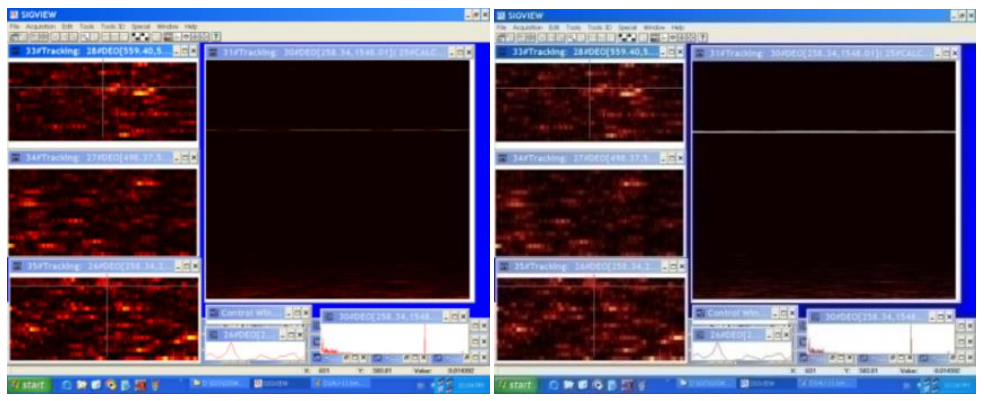

Figure 4

EEG recordings of inner music. Left: Parts of spectrograms of EEG channels with recording of inner (imagined) $\mathrm{d} 2$ tone; right: extracted inner tone in the right window in a composite spectrogram, shown enhanced bottom - right.

The automatic fuzzy classifier measures the degree of similarity with the precalibrated fuzzy tones, picking the best match (presented in $[9,13]$ ). The method, based on local linear dependence and comparison to the set of special calibrated sets of adjustable fuzzy filters for more general application, is being further developed. Here, we have fuzziness at both modeling and intelligence levels. For some applications smoothing/roughening of the features is needed; these operations correspond to a variety of defuzzyfications.

\section{Example 2}

In the following important examples of the arterial blood pressure (BP), we have further feature invariant determination; spectrogram features with relevant details are shown in Fig. 5. The exhibited morphology provides for different ways of capturing the characteristics of relevant features (the steps described above). In the top left picture, we can see tracing of the linear inclination of the high frequency 
feature whose non-homogenous morphology is shown in the lower window. Second order spectrum of the top feature longitudinal section is its essential invariant. On the right is displayed a BP spectrogram of features with their photometric representations, demonstrating the level of the noise surrounding these features and the problems related to the contour definition. These important patterns need a mathematically precise definition and characterization. Again, the (second order) spectrogram of the top left structure longitudinal section is its essential invariant, identical to the same invariant of the feature on the right. Here, pattern similarity matching is available as matching of the dominant lines in the corresponding second order power spectra.

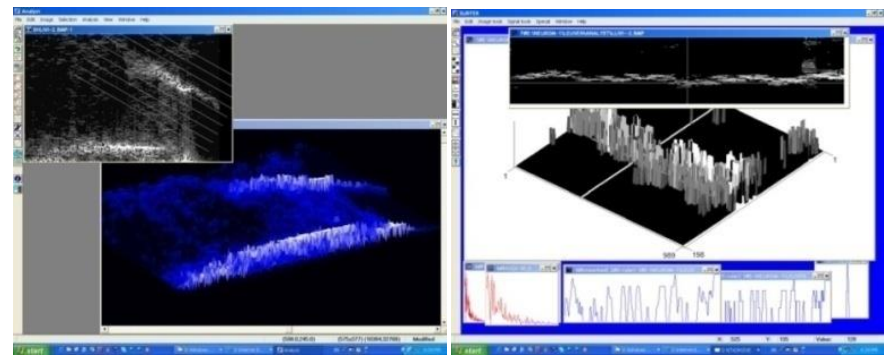

Figure 5

Recording of the blood pressure - BP; Features in the spectrograms with their photometric representations

\section{Example 3}

Illustrations of the performance of discussed steps in the automatic feature recognition are shown in Figs. 6-8. For the investigation of some dynamic feature characteristics, e.g. those related to both time/rhythmic and frequency changes, or metric invariants, or those involving second order spectra, the analysis of features in their original shape, are necessary, together with some normalized form. Figure 6 shows an example of the step-by-step background noise elimination on the left. The automatic algorithm is based on threshold optimization: increasing the threshold until the image is fragmented into objects with larger than a parametric diameter and surface, subsequently reducing the threshold to enlarge detected objects till the level of surrounding noise increases above a parameter, while zeroing noncontiguous dot clusters. The automatic object contour definition is shown on the right, followed by the topology tracings of individual objects, which in turn is followed by the normalization based on its topologic structure. The normalization is necessary for the invariants determination and comparisonsmatching. Originally developed for the structural study of microscopic images of chromosomes, this algorithm is adapted for time spectral feature localization, extraction and some normalization with feature comparison. 

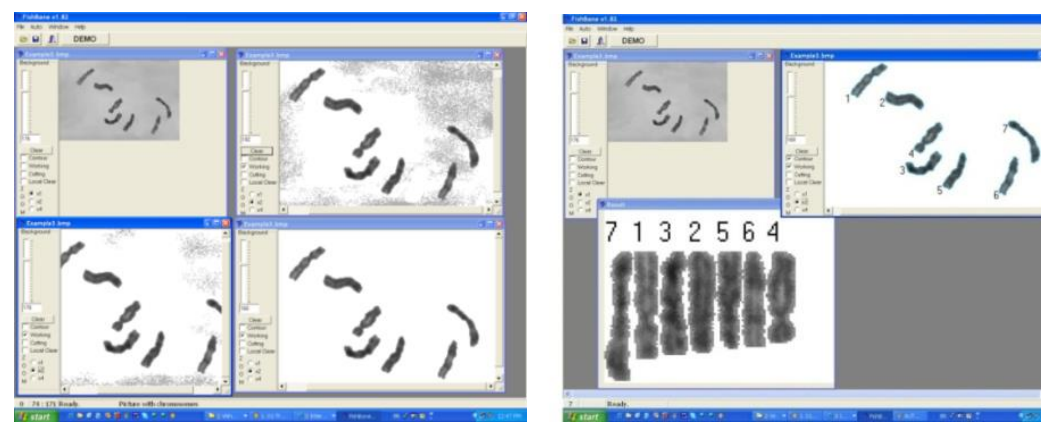

Figure 6

Left: background noise elimination; right: Automatic object contour definition
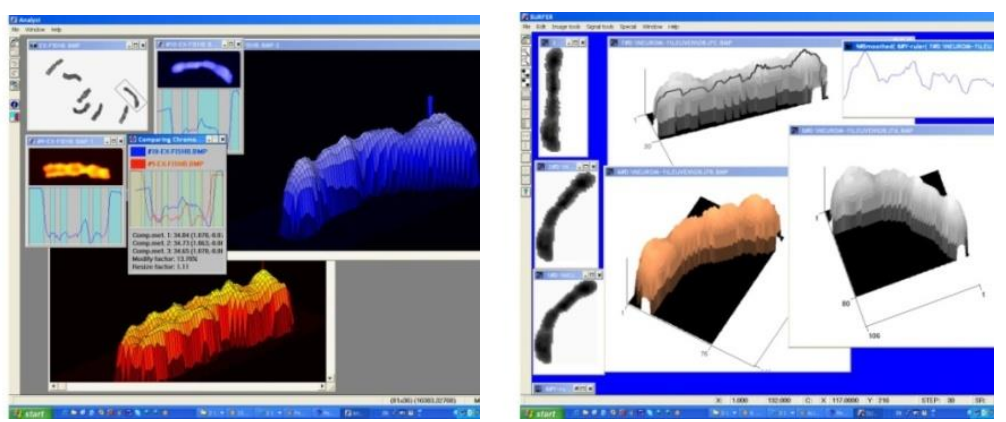

Figure 7

Left: The feature normalization and automatic comparison. Right: Feature step by step normalization with longitudinal sections exhibiting changes in morphology - dynamics.

\section{Example 4}

One example of an acoustic spectrogram with locally well-defined and wellseparated features, which are processed through the steps described above, is given in Fig. 8.

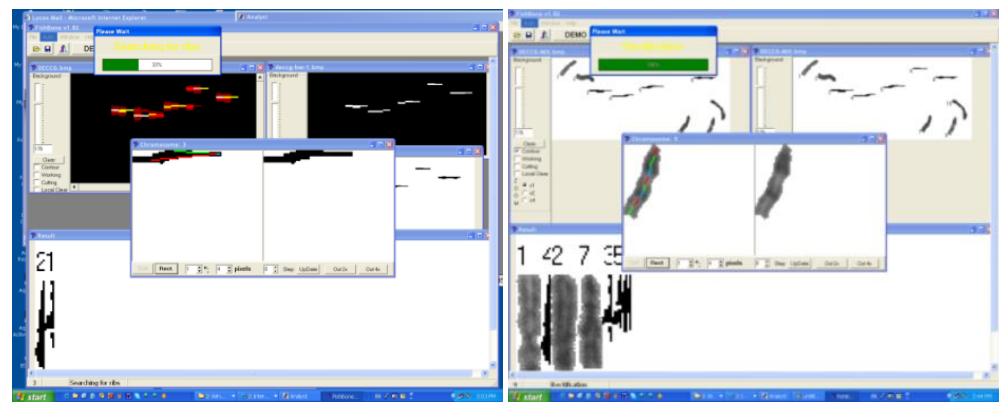

Figure 8

An automatic real time feature recognition; Synthetic spectrogram 
Left: The real time spectrogram of a simple melody (deccg) with the automatic feature recognition; defuzzification was applied first, followed by normalization and the measurement of flexure angles and object extraction (lower). On the right, we have the same spectrogram enriched with a number of contiguous curved structures, non-constant in frequency, subjected to the same algorithm for the automatized feature recognition and classification, an adaptation of algorithm originally developed for automatic recognition of chromosomes in CCDmicroscopic images.

\subsection{Small Object Recognition}

In this section we will give a brief overview of an alternative method for the efficient recognition of smaller, dot-like objects with the diameter $<10$ pixels. Method can be applied to both matrices and vectors. Short frequency pulses are an important example of these. Spectral features which are stable and narrow in frequency might be examples of such sorts of vectors. Previously, we developed procedures for small object recognition and filtering by size based on the intensity discrimination (intensity of considered pixels). The method we present here is an improved Tomasi, Shi, Kanade procedure for the extraction of the characteristic features from a bitmap image (see [11] and [15]). It is robust and proved to be efficient, possessing all highly desirable properties, as illustrated in the subsequent figures. As an input we have a simple monochrome $(0=$ white, $255=$ black $)$ bitmap (matrix) $A$ of a fixed format (here presented with $400 \times 400$ pixel resolution). The components of $A$ signal amplitude values, or e.g. spectrogram intensities, will be denoted by $A(x, y)$, where $x$ indicates the corresponding row and $y$ indicates the corresponding column. Spatial $x$-wise and $y$-wise differences $I_{x}$ and $I_{y}$ are defined as follows:

$$
I_{x}=\frac{A(x+1, y)-A(x-1, y)}{2}, \quad I_{y}=\frac{A(x, y+1)-A(x, y-1)}{2}
$$

The matrix $G$ of sums of spatial square differences is defined by

$$
G=\sum_{x=p_{x}-\omega_{x}}^{p_{x}+\omega_{x}} \sum_{y=p_{y}-\omega_{y}}^{p_{y}+\omega_{y}}\left[\begin{array}{cc}
I_{x}{ }^{2} & I_{x} I_{y} \\
I_{x} I_{y} & I_{y}{ }^{2}
\end{array}\right] .
$$

where $\omega_{x}=\omega_{y}$ is the width of the integration window (the best results are obtained with values between 2 and 4), while $p_{x}$ and $p_{y}$ are the indices corresponding to the indices $x$ and $y$ such that the formula (2) is defined; therefore, all inner pixels (i.e. pixels for which $I_{x}$ and $I_{y}$ can be defined) are included in the computation. We rewrite $G$ in the more compact form as

$$
G=\left[\begin{array}{ll}
a & b \\
c & d
\end{array}\right]
$$

Using the above compact form (3) of $G$ we can compute its eigenvalues as 


$$
\lambda_{1,2}=\frac{a+d}{2} \pm \frac{\sqrt{(a-d)^{2}+4 b c}}{2} .
$$

Furthermore, for each inner pixel with coordinates $(x, y)$ we define $\lambda(x, y)$ by

$$
\lambda(x, y)=\min \left(\lambda_{1}(x, y), \lambda_{2}(x, y)\right) .
$$

Finally, for the given lower threshold $T_{\min }$, the parameter $A_{\max }$ (in our examples $A_{\text {max }}$ is equal to 255 ) set the value

$$
\lambda_{\max }=\max \{\lambda(x, y) \mid(x, y) \text { is an inner pixel }\} .
$$

We define the extraction matrix $E$ by

$$
E(x, y)=\left\{\begin{array}{ll}
\frac{A_{\max }}{\lambda_{\max }} \cdot \lambda(x, y), & \frac{A_{\max }}{\lambda_{\max }} \cdot \lambda(x, y)>T_{\min } \\
0, & \frac{A_{\max }}{\lambda_{\max }} \cdot \lambda(x, y)<T_{\min }
\end{array},\right.
$$

When two images or spectrograms are available (two consecutive shots or two significantly linearly independent channels) we obtain a solution in an even harder case for automatic extraction. Let $B$ and $C$ be two images where every pixel is contaminated with noise which has a normal Gaussian distribution, in which a stationary signal is injected, objects at coordinates $\left(x_{1}, y_{1}\right), \ldots\left(x_{10}, y_{10}\right)$, all with an intensity of e.g. $m$ (within $[0,255]$ interval) and fluctuation parameter $p$; we generate the new binary image $A$ in two steps:

$$
\begin{gathered}
A(x, y)=\operatorname{abs}(B(x, y)-C(x, y)) \\
\text { If } A(x, y)<p \text { then } A(x, y)=255 \\
\text { else } A(x, y)=0 ;
\end{gathered}
$$

The above simple discrimination reduces random noise significantly and reveals the signals together with residual noise. By performing the procedure defined by the equations (1) thru (7), we obtain the filtered image with extracted signals. The method is adaptable, using two parameter optimization (minimax): the minimalization of the integral surface of detected objects, then the maximization of the number of the small objects.

An alternative method for the detection/extraction of small features is based on a bank of Kalman filters. After the construction of the initial sequence of images, $Z_{k}$, the bank of one-dimensional simplified Kalman filters (see e.g. [16]) is defined using the iterative procedure as follows:

$$
\begin{gathered}
K_{k}(x, y)=\frac{P_{k-1}(x, y)+Q}{P_{k-1}(x, y)+Q+R} \\
\hat{X}_{k}(x, y)=\hat{X}_{k-1}(x, y)+K_{k}(x, y) \cdot\left(Z_{k}(x, y)-\hat{X}_{k-1}(x, y)\right) \\
P_{k}(x, y)=\left(1-K_{k}(x, y)\right) \cdot\left(P_{k-1}(x, y)+Q\right) .
\end{gathered}
$$


Initially, $P_{0}(x, y)=\hat{X}_{0}(x, y)=0, Q=1, R=100$, where $Q$ is the covariance of the noise in the target signal and $R$ is the covariance of the noise of the measurement. Depending on the dynamics of the problem we put: the output filtered image in $k^{\text {th }}$ iteration is the matrix $\hat{X}_{k}$, the last of which is input in the procedure described by equations (1) to (7), finally generating the image with the extracted objects.

This method shows that it is not necessary to know the signal level if we can estimate the statistical parameters of noise and statistics of measured signal to some extent. In the general case, we know that its mean is somewhere between 0 and 255 and that it is contaminated with noise with the unknown variance.

The method of small object recognition, originally developed for the marine radar object tracking, works with vectors equally well. It is applicable to the automatic extraction of signals which are embedded in the noise and imperceptible (also in the spectra) in the case when we can provide at least two sources which are sufficiently linearly independent (their linear dependence on the signal components is essential for the object filtering - extraction), or in the situations when the conditions for application of Kalman filters are met.

\section{Example 5}

In this example, we have introduced several dots (useful signals) with an amplitude of $a=120$, and we have contaminated the image with random and cloudlike noise. The image on the left in Fig. 9 shows a bitmap with random contamination of the signal - dots. The image on the right in the same figure shows the resulting bitmap after the application of the procedure for noise reduction. After the initial setting $A_{\max }=255$ and $T_{\min }=124$, the extraction procedure yields the image shown below in Fig. 10.

The image on the left in Fig. 11 shows a similar example of the signal - dots contaminated with a cloudy noise containing granular elements which are similar in size and intensity to the signal. The image on the right shows the results of the reduction of noise: some new dots belonging to the noise cannot be distinguished from the signal - top and low right.

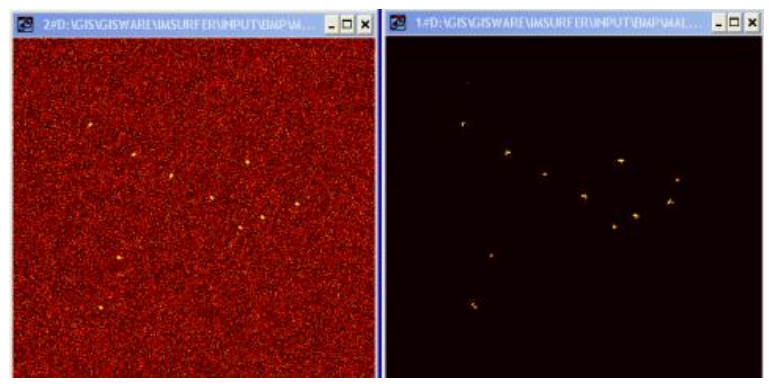

Figure 9

Reduction of noise 


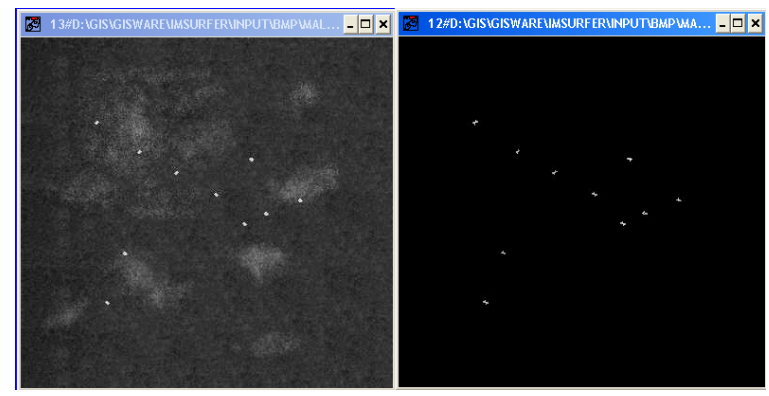

Figure 10

left: signal - dots, contaminated with cloudy noise; right: extraction of signal.

Note that the amplitude of the target signal is lower than the chosen lower threshold (images in Figs. 9 to 11).

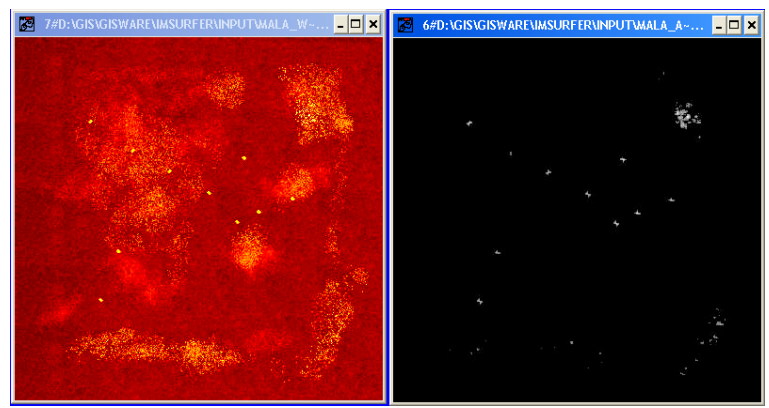

Figure 11

Signal extraction

\section{Example 6}

Here we illustrate the application of the method of small feature extraction with two independent sources, shown in Fig. 12, with the signals embedded in the noise and the process of signal extraction.

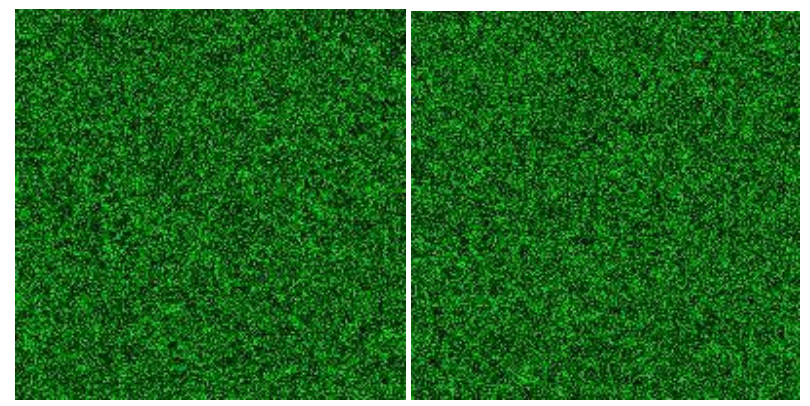

Figure 12

Two Gaussian noise images with the injected small objects below threshold 


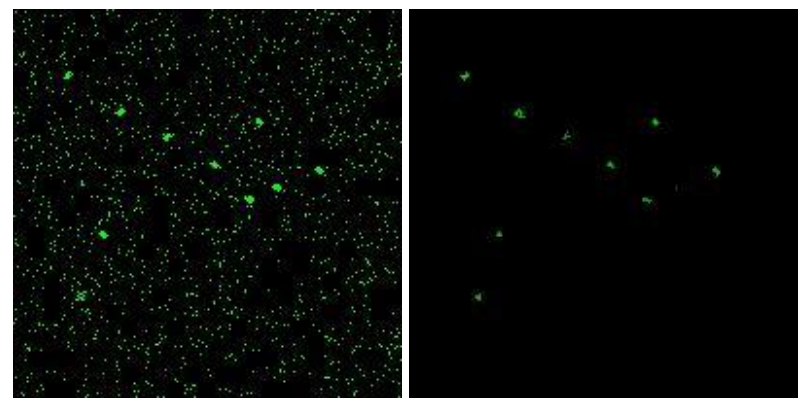

Figure 13

Left: Extraction of the objects based on simple discrimination. Note the presence of residual noise smaller dots. Right: after application of the above method to the binary image on the left, the noise is completely reduced, yielding fully automatic small object recognition.

\section{Example 7}

The application of Kalman filters in small object extraction. In the experiment shown, the initial sequence of images, $Z_{k}$, of the size $200 \times 200$ pixels is generated as follows. First, in each image we have introduced noise by $Z_{k}(x, y)=$ randn $(0,90)$; here "randn" generates random numbers in the interval $[0,255]$ using Gaussian distribution with $\mu=0$ and $\sigma=90$. Then, in every image we injected 10 objects (useful signals) at the same positions, each of them of a size around 10 pixels, with random (Gaussian) fluctuation of intensity around value 120. After the construction of the initial sequence of images, the bank of $200 \times$ $200=40000$ one-dimensional simplified Kalman filters is defined using the iterative procedure as above. The process of noise elimination and feature extraction is shown in the images in Fig. 15.
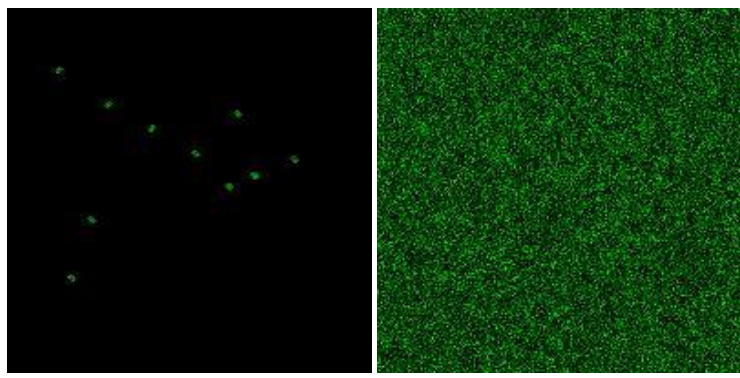

Figure 14

Left: Injected signal; Right: The image on the left injected with the Gaussian noise contamination. 

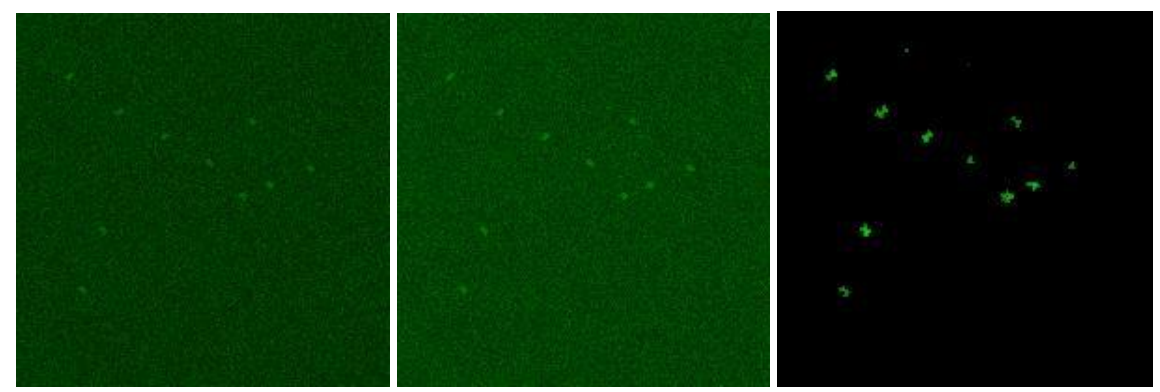

Figure 15

Left: The result of processing after the $21^{\text {st }}$ iteration of Kalman filter banks: Center: The result of processing after 34 iterations; Right: The result of processing after 36 iterations and application of the method described by formulae $1-8$, providing a complete object extraction.

\section{Example 8}

Localization and extraction of the small size features in spectrograms of diverse origin. In Fig. 16 left and center, we show examples from [17] (similar examples are widely distributed in literature), which are used in brain connectivity pattern detection. The resolution here is: pix $=2 \mathrm{~Hz} * 0.5 \mathrm{~s} ; 2 * 1 ; 2 * 2$. Note the size of the granulae in the shown spectrograms. The great majority of the important features are within $6 \times 6$ pix, and the method of small object recognition performs very well even with some noise contamination. In Figure 16, on the right is shown our RT reproduction (whistling) of the melody used by A. Ioannidis in his impressive presentation of MEG tomography, with the same melody stimulation (available on his site as well); all spectrogram granulae are within $4 \times 4$ pix size (Easy to generate with the available DEMO at our site). Good examples for the application of this method are spectrograms in the second, third and fourth quadrant in Fig. 3 left and center; in the presented context this method can be performed concurrently with the method from section 2.1 for parallel recognition of larger structures, as are those shown in the first quadrants of these images and features in the image on the right. The same is true in the case when both types of structures are overlapping, as in Fig. 17 with FISH signals. The extraction of the small objects within the cloudy structures in conjunction with the earlier described method based on the contour detection provides a means for the automated and concurrent detection of small and large structural components independently. 

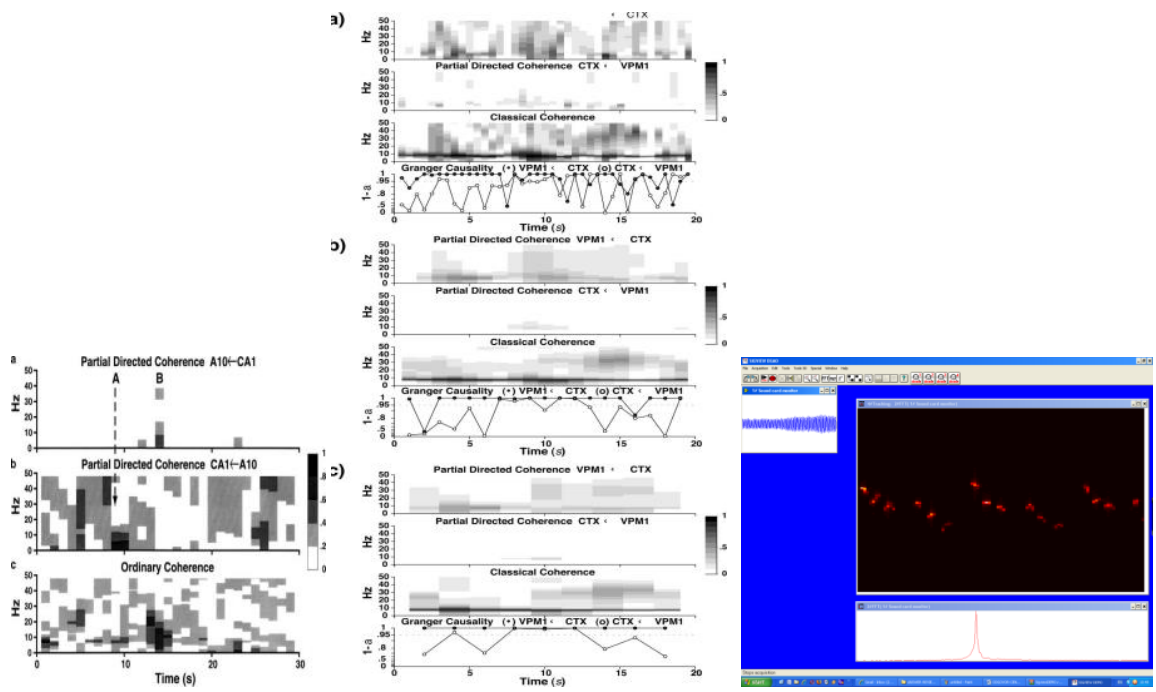

Figure 16

Left and center: brain connectivity relevant spectrograms from [17]: frequency range $50 \mathrm{~Hz}$, time $35 \mathrm{~s}$, granular dimensions easy readable, Resolution: pix $=20 \mathrm{~Hz} * 4 \mathrm{~s}$; a number of small size spectrogram objects are in the size of up to $5 \times 5$ pixels. Right: RT reproduction of A. Ioannides MEG example with spectrogram consisting of small features.

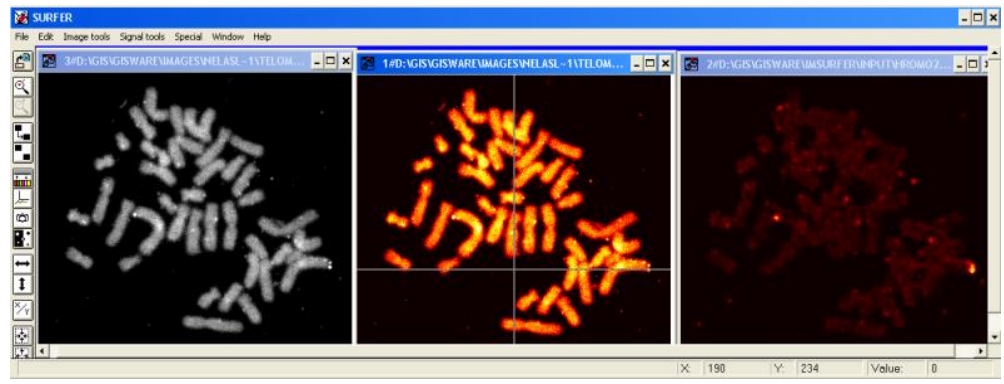

Figure 17

Extraction of FISH signals from chromosomes with the same method

\section{Conclusion and Discussion}

This paper addresses the problem of the automatized recognition of features in signals and their Fourier or wavelet spectra and spectrograms. The algorithms presented use techniques developed for image processing and are suitable for morphologic investigations. These algorithms are able to localize and extract features and to determine their topologic and geometric characteristic invariants, which are used to represent and to classify the objects by applying subtle similarity measures. Small object recognition in cases of heavy contamination by 
noise of mainly random nature is successfully performed in rather general circumstances. This is applied well to the automatic recognition of short frequency pulses in the spectrograms. The methods presented are useful even for semiautomated or manual cases, when for example their automated application is limited because of some of the discussed reasons and can be applied for a detailed structural inspection and comparison of the features. Due to a modest complexity, all are real time (RT) applicable, even without the enhanced hardware.

Obviously, the real experimental practice always offers nice counterexamples that do not fit well into the predefined conceptual scheme, such as parts of the features in the BP spectrograms at the top of Fig. 5 (or granular noise indistinguishable from the objects - dots in Fig. 15). Photo morphology revealing the lower feature contour is very fuzzy. The left part of the top structure can hardly be called a feature at all, but rather a random cloud of dots. But the complete set of the dots is definitely functionally related. We have the appearance of a feature out of the randomness, which characterizes some micro-phenomena that are still not semantically bound at the macroscopic scale. This is a kind of reality where the method presented here might exhibit some problems. In circumstances like this, one should search for transformations that are able to convert information in the signal into simpler topological or geometrical structures. Nevertheless, our approach can be well applied to a variety of different cases of real time spectrogram features. Similarity and pattern classification in the continuous domains is properly modeled with metrics in the classic metric spaces, and the majority of our implemented similarity measures are of this kind. There are many other approaches. One mentioned earlier is the recognition of bumps in EEG spectra [18], which is done in the similar spirit as the perspective of this paper. The brain is investigated with nonlinear analysis methods too. The chaos theory is applied in the analysis of brain activity; the estimation of the fractal dimension in time domain gives a measure of signal complexity. It has been successfully used with some brain injuries [19].

Finally, it is worth mentioning that the theoretical development and steadily growing applications of pseudo-analysis are giving alternative methods for the mathematical design of the extraction criteria, automatic threshold design and so on; see for instance [20, 21].

\section{Acknowledgements}

This research has been partly supported by Serbian Ministry of Education and Science, projects III-41013, TR36001 and ON174009.

\section{References}

[1] A. Jovanović, $C D-R O M$ : $C C D$ Microscopy, Image \& Signal Processing, (School of Mathematics, Univ. of Belgrade, Belgrade, 1997)

[2] A. Jovanović, A. Perović, Brain Computer Interfaces - Some Technical Remarks, International Journal of Bioelectromagnetism 9(3): 91-102, 2007 
[3] F. Cincotti, D. Mattia, C. Babiloni, F. Carducci, L. del R. M. Bianchi, J. Mourino, S. Salinari, M. G.Marciani, F. Babiloni, Classification of EEG Mental Patterns by Using Two Scalp Electrodes and Mahalanobis Distance-based Classifiers, Method of Information in Medicine 41(4) (2002) 337-341

[4] G. Pfurtscheller, C. Neuper, C. Guger, W. Harkam, H. Ramoser, A. Schlög, B. Obermaier, and M. Pregenzer, Current Trends in Graz Brain-Computer Interface (BCI) Research, IEEE Transaction on Rehabilitation Engineering 8(2): 216-219, 2000

[5] F. Nijboer, E. W. Sellers, J. Mellinger, M. A. Jordan, T. Matuz, A. Furdea, S. Halder, U. Mochty, D. J. Krusienski, T. M.Vaughan, J. R. Wolpaw, N. Birbaumer, A. Kübler, A P300-based Brain-Computer Interface for People with Amyotrophic Lateral Sclerosis. Clinical Neurophysiology 119(8): 1909-1916, 2008

[6] A. Jovanović, Brain Signals in Computer Interface, (in Russian, Lomonosov, Russ. Academy of Science), Intelektualnie Sistemi, 3(1-2): 109-117, 1998

[7] R. J. Zatorre, A. R. Halpern, Mental Concerts: Musical Imagery and Auditory Cortex, Neuron, 47: 9-12, 2005

[8] J. K. Kroger, L. Elliott, T. N. Wong, J. Lakey, H. Dang, J. George, Detecting Mental Commands in High-Frequency EEG: Faster BrainMachine Interfaces, in Proc. 2006 Biomedical Engineering Society Annual Meeting, (Chicago, 2006)

[9] Wlodzimierz Klonowski, Wlodzisław Duch, Aleksandar Perovic, and Aleksandar Jovanovic, "Some Computational Aspects of the Brain Computer Interfaces Based on Inner Music," Computational Intelligence and Neuroscience, Vol. 2009, Article ID 950403, 9 pages, 2009. doi:10.1155/2009/950403

[10] G. Pfurtscheller, F. H. Lopes da Silva, Event-related EEG/MEG Synchronization and Desynchronization: Basic Principles, Clinical Neurophysiology, 110(11): 1842-1857, 1999

[11] J. Y. Bouguet, Pyramidal Implementation of the Lucas Kanade Feature Tracker, http://robots.stanford.edu/cs223b04/algo_affine_tracking.pdf

preprint,

[12] G. Bradski, Computer Vision Tracking for Use in a Perceptual User Interface, http://www.cse.psu.edu/ rcollins/CSE598G/papers/camshift.pdf

preprint,

[13] S. Spasić, A. Perović, W. Klonowski, Z. Djordjević, W. Duch, A. Jovanović, Forensics of Features in the Spectra of Biological Signals, International journal for bioelectromagnetism 12(2): 62-75, 2010 
[14] A. Jovanović, A. Perović, W. Klonowski, W. Duch, Z. Đorđević, S. Spasić, Detection of Structural Features in Biological Signals, Journal of Signal Processing Systems 60(1): 115-129, 2010

[15] J. Shi, C. Tomasi, Good Features to Track, preprint, http://www.ai.mit.edu/courses/6.891/handouts/shi94good.pdf

[16] G. Welch, G. Bishop, An Introduction to the Kalman Filter, University of North Carolina at Chapter Hill, Chapter Hill, TR 95-014, April 5, 2004

[17] K. Sameshima, L. A. Baccala, Using Partial Directed Coherence to Describe a Neuronal Assembly Interactions, J. Neurosci Methods 94: 93103, 1999

[18] F. B. Vialatte, J. Sole-Casals, J. Dauwels, M. Maurice and A. Cichocki, Bump Time-Frequency Toolbox: a Toolbox for Time-Frequency Oscillatory Bursts Extraction in Electrophysiological Signals, BMC Neuroscience 10(46), doi:10.1186/1471-2202-10-46, 2009

[19] S. Spasić, M. Culić, G. Grbić, Lj. Martać, S. Sekulić, D. Mutavdžic, Spectral and Fractal Analysis of Cerebellar Activity after and Repeated Brain Injury, Bulletin of Mathematical Biology 70 (4): 1235-1249, 2008

[20] E. Pap. Pseudo-Analysis Approach to Nonlinear Partial Differential Equations. Acta Polytechnica Hungarica 5(1): 30-44, 2008

[21] E. Pap, V. Bojanić, M. Georgijević, G. Bojanić. Application of PseudoAnalysis in the Synchronization of Container Terminal Equipment Operation. Acta Polytechnica Hungarica 8(6): 5-21, 2011 